\title{
Atrial Fibrillation Detection Using Stationary Wavelet Transform and Deep Learning
}

\author{
Yong Xia ${ }^{1}$, Naren Wulan ${ }^{1}$, Kuanquan Wang ${ }^{1}$, Henggui Zhang ${ }^{1,2}$ \\ ${ }^{1}$ Harbin Institute of Technology, Harbin, China \\ ${ }^{2}$ University of Manchester, Manchester, United Kingdom
}

\begin{abstract}
Deep learning has achieved a great success in the fields of image and audio recognition because of avoiding explicit feature extraction and attaining high classification accuracy. In this paper, we explore the application of deep convolutional neural networks (DCNNs) for automatic detection of atrial fibrillation $(A F)$. The 2-dimension parameter input structure is essential for DCNNs and tens of thousands of samples are also needed for the proper operation. As we know, ECG is one-dimension time-varying signal, which doesn't match the requirement for the input structure of DCNNs. Furthermore the number of the marked AF samples is also limited. To address these problems, we adopt the stationary wavelet transform (SWT) for ECG preprocessing and then the processed signal is reorganized into two-dimensional parameter structure to meet the requirement of input structure of DCNNs. Besides, the original ECG signals are divided into very short data segments (namely 5-second segments) for the following reasons. On the one hand, short ECG segment is helpful for the algorithm assessment of short $A F$ episode detection. On the other hand, it can also increase the number of $A F$ sample for machine learning and experiment evaluation. As for DCNNs, multiple convolutional layers and fully connected layers are used for deep learning. On the MIT-BIH Atrial fibrillation data set, the proposed method can achieve sensitivity of $98.79 \%$, specificity of $97.87 \%$ and accuracy of $98.63 \%$, which outperforms most of other algorithms.
\end{abstract}

\section{Introduction}

Atrial fibrillation (AF) is the most common cardiac arrhythmia. Though AF itself does not represent a lethal condition, it can increase risks of morbidity or even mortality due to AF-related complications. It has been reported that the presence of AF is associated with a 3fold risk of developing heart failure (HF) [1] independent of other risk factors.

Currently, a wide variety of algorithms have been developed for automatic AF detection. These algorithms mostly rely on the absence of P-waves or irregularity of
$\mathrm{R}-\mathrm{R}$ intervals or some hand-crafted features [2]. The performance of most extant algorithms strongly depends on the detection of $\mathrm{P}$ or $\mathrm{R}$ peak. If the related peaks are missed or erroneously detected, their performance will substantially degrade [3]. Furthermore, due to the challenges of extracting reliable feature, the methods needed hand-crafted feature extraction may not fit to be widely applied clinically. Aimed at addressing the deficiencies and drawbacks of existing $\mathrm{AF}$ detection algorithms, we propose a novel method for automatic AF detection based on deep convolutional neural networks (DCNNs).

Deep learning develops a computational models consist of multiple processing layers which can learn abstract representations (or called feature maps) of data [4]. Deep learning allows a machine to take raw data as input and to automatically discover the representations needed for detection or classification. For discovering intricate structure in data sets, deep learning transforms the representation of one level to the representation of a higher and abstract level through a non-linear function. And then it uses the back propagation algorithm to change parameters which are used to calculate the representation of each layer. After enough of such transformations and changes, a machine can learn a complex function for feature extraction and classification [5]. Over passed years, deep learning has been proved very successful in speech recognition, image recognition and many other domains [5]. However, deep learning has not been used widely in ECG analysis and classification because of small training collection and specificity of ECG signal. How to build an effective learning strategy for ECG signal is still a challenging problem.

To our knowledge, this is the first study using DCNNs for the purpose of AF detection. As opposed to traditional approaches, the proposed approach does not require $\mathrm{P}$ or $\mathrm{R}$ peak detection and voids the need for any manual feature extraction. With proper training, the convolutional layers of DCNNs can learn to extract patient-specific features.

\section{Methods}

\subsection{Pre-processing}


The original beat-to-beat annotated ECG signal is divided into segments for every 5 seconds by using a percentage parameter $\mathrm{P}$ [6]: A segment is considered to be a true AF only when the annotated AF beats in that data segment with a percentage greater than $\mathrm{P}$. In our study, we set $\mathrm{P}$ to 0.5 . In order to remove baseline wander, muscle noise and power-line interference, an elliptical band-pass filter with filter order of 10 and passband of 0.5-50 Hz is applied to each segment.

\subsection{Stationary wavelet transform}

We choose the stationary wavelet transform (SWT) since it is time-invariant at each decomposition level. Since the SWT with J-levels on a signal requires the signal length to be a multiple of $2^{\mathrm{J}}$, the filtered data segment needs to be zero-padded. The detail coefficients $D_{j}(n)$ and coarse coefficients $C_{j}(n)$ in time domain can be recursively computed as:

$$
\begin{aligned}
& D_{j}(n)=\sum_{m} g(n) D_{j-1}\left(n-2^{j-1} m\right) \\
& C_{j}(n)=\sum_{m} h(n) C_{j-1}\left(n-2^{j-1} m\right)
\end{aligned}
$$

After pre-processing step, we employ SWT on each 5second segment to obtain one kind of representation on filtered data. It is worth noting that we use SWT to generate $2 J$ ( $J$ detail coefficients and $J$ coarse coefficients) time series for each segment. Each coefficient time series has the same time resolution as the original signal segment. The $2 \mathrm{~J}$ time series are organized in a two-dimensional (2-D) matrix of which every row stands for one coefficient time series. DCNNs are often used for image classification, and require the 2D array input. For color images, RGB (red, green, blue) values can be regarded as three different 2-D feature maps. The 2-D matrix of coefficients time series can be regarded as a grayscale "image". Thus, this type of representations can be used as 2-D input of DCNNs. Figure 1 shows the 2-D matrix of coefficients time series input type normalized to $[-1,1]$. As mentioned above, the 2-D matrix of coefficients time series is the $2 \mathrm{~J}$ time series where $J$ means SWT with J-levels. In this study, we choose the Daubechies 5 wavelet as the mother wavelet, which is an orthogonal wavelet and is similar to the ECG waveform [7], to implement wavelet analysis. Considering the sampling frequency of $250 \mathrm{~Hz}$ and the frequency range of atrial activities $(4-9 \mathrm{~Hz})$ [8], we set the number of wavelet transform decomposition level $J$ to 6 . So Figure 1 has total 12 rows (6 rows of detail coefficients and 6 rows of coarse coefficients).

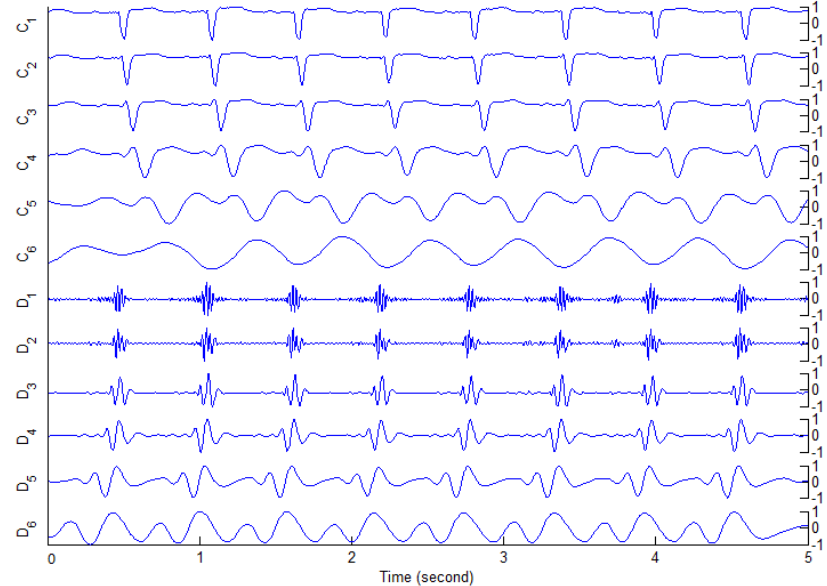

Figure 1. Graphical representation of a 5-second 2-D matrix of coefficients time series used as the input of DCNNs.

\subsection{DCNNs architectures}

DCNNs are a popular type of deep learning architecture and are composed of mainly three types of layers: convolutional layers, pooling layers and fully connected layers. A convolutional layer applies a set of weights called filter bank or kernel to process small local parts of the feature map from previous layer (or raw input). Feature maps consist of many neurons called units. Each unit in feature maps of current convolutional layer is connected to local areas in the feature maps of the previous layer through the filter bank. The sum of the local weights is then passed through a non-linear function such as a ReLU. It is noteworthy that in a convolutional layer, different feature maps use different filter banks but all units of one feature map share the same filter bank. The convolutional layer plays the role of detecting local connections of features from the previous layer but the pooling layer plays the role of merging similar features into one. Due to neighbouring units have correlation with each other, reliable detection can be done by generating a lower resolution feature map. Pooling layer can reduce the dimension of feature maps and the number of parameters, and create an invariance to translation and distortion. At the end of the DCNNs, there are usually some fully connected layers. Top fully connected layers perform the classification task and produce the final class vector. The back propagation algorithm used in the DCNN adjusts all the weights in all filter banks as it does in a conventional deep network. In brief, there are four main concepts behind the DCNNs: local connections, sharing weight, pooling and the use of multiple layers.

Table 1 shows the detailed architecture of DCNNs for automatic AF detection. The architecture is composed of two convolutional layers, two max-pooling layers, two ReLU layers, one dropout layer, two fully connected 
layers and one softmax layer. The first convolutional layer with kernel size of $3 \times 11$ has 32 hidden units followed by a ReLU layer. The pooling size of the first pooling layer is $2 \times 3$. The second convolutional layer with kernel size of $2 \times 11$ has 32 hidden units. The pooling size of the second pooling layer is $2 \times 3$. The first fully connected layer has 100 hidden units followed by a ReLU layer and then a dropout layer. The second fully connected layer has 2 hidden units. Finally, there is a softmax layer with 2 outputs.

Table 1. The detailed architecture of DCNNs.

\begin{tabular}{ccc}
\hline Layer & $\begin{array}{r}\text { \# of } \\
\text { output }\end{array}$ & $\begin{array}{c}\text { Kernel size/ } \\
\text { Pooling size }\end{array}$ \\
\hline Conv1 & 32 & $3 \times 11$ \\
ReLU1 & 32 & \\
Pool1 & 32 & $2 \times 3$ \\
Conv2 & 32 & $2 \times 11$ \\
Pool2 & 32 & $2 \times 3$ \\
Fc1 & 100 & \\
ReLU2 & 100 & \\
Dropout1 & & \\
Fc2 & 2 & \\
Softmax & 2 & \\
\hline
\end{tabular}

\section{Results}

\subsection{Data set}

The MIT-BIH Atrial fibrillation (MIT-BIH AFIB) data set [9] which is the most popular database for the assessment of AF detection is used to evaluate the performance of the proposed algorithm.

\subsection{Experimental result}

The experiments are performed on a computer with 2 CPU at 2.1 GHz , 2 NVIDIA Tesla K40c GPU and 32-Gb memory. We run the proposed DCNNs over our highly efficient GPU using Caffe deep learning framework [10]. Table 2 summarizes the performance of our method and the existing algorithms [2, 11-13] published in recent few years validated on the MIT-BIH AF database.

Huang[11] and Lee[12] methods need to detect R-R intervals. Babaeizadeh[13] methods need to detect both $\mathrm{R}-\mathrm{R}$ intervals and $\mathrm{P}$ wave. Their performances depend on the accuracy of peak detection and when missing peaks or erroneously detected peaks, their performance will be poor. Although, Asgari[2] method does not need to detect $\mathrm{R}-\mathrm{R}$ intervals or $\mathrm{P}$ wave, they manually selected the peakto-average power ratio and log-energy entropy of the signal as features of AF detection.

In contrast, our proposed method does not rely on peak detection and other manual features and achieves the sensitivity of $98.79 \%$, specificity of $97.87 \%$ and accuracy of $98.63 \%$, which outperform all other methods from Table 2.

Table 2. The comparison of performance.

\begin{tabular}{lll}
\hline \multicolumn{1}{c}{ Algorithm } & Sensitivity & Specificity \\
\hline Huang et al.[11] & $96.10 \%$ & $98.10 \%$ \\
Lee et al.[12] & $98.20 \%$ & $97.70 \%$ \\
Babaeizadeh et al.[13] & $92.00 \%$ & $95.50 \%$ \\
Asgari et al.[2] & $97.00 \%$ & $97.10 \%$ \\
Our method & $\mathbf{9 8 . 7 9 \%}$ & $\mathbf{9 7 . 8 7 \%}$ \\
\hline
\end{tabular}

\section{Conclusion}

In this paper, we convert the one-dimensional ECG signal into two-dimensional form by stationary wavelet transform, so as to realize the detection of atrial fibrillation using deep convolution neural network. In this way, there is no need for the detection of P or R peak and the extraction of handcrafted manual feature. The results of experiments, which are performed over the MIT-BIH Atrial fibrillation (MIT-BIH AFIB) database show that our DCNNs with input form based on SWT has sensitivity of $98.79 \%$, specificity of $97.87 \%$, accuracy of $98.63 \%$, which outperforms the majority of the existing algorithms. Furthermore, the proposed method performs very well for data segment as short as 5-second. As a result, the proposed approach is a fast, accurate, efficient method for detection of atrial fibrillation.

\section{Acknowledgments}

This work was financially supported by Shandong Province Natural Science Foundation (ZR2015FM028), Shandong Province Science and Technology Development Plan (2014GSF118152), and China Scholarship Council (201606125090).

\section{References}

[1] Camm A J, Lip G Y, De Caterina R, et al. ESC Committee for Practice Guidelines (CPG). 2012 focused update of the ESC Guidelines for the management of atrial fibrillation: an update of the 2010 ESC Guidelines for the management of atrial fibrillation. Developed with the special contribution of the European Heart Rhythm Association[J]. Eur Heart J, 2012, 33(21): 2719-2747.

[2] Asgari S, Mehrnia A, Moussavi M. Automatic detection of atrial fibrillation using stationary wavelet transform and support vector machine[J]. Computers in biology and medicine, 2015, 60: 132-142.

[3] Larburu N, Lopetegi T, Romero I. Comparative study of algorithms for atrial fibrillation detection[C]//Computing in Cardiology, 2011. IEEE, 2011: 265-268.

[4] Goh H, Thome N, Cord M, et al. Learning deep 
hierarchical visual feature coding[J]. IEEE transactions on neural networks and learning systems, 2014, 25(12): 2212-2225.

[5] LeCun Y, Bengio Y, Hinton G. Deep learning[J]. Nature, 2015, 521(7553): 436-444.

[6] Dash S, Chon K H, Lu S, et al. Automatic real time detection of atrial fibrillation[J]. Annals of biomedical engineering, 2009, 37(9): 1701-1709.

[7] Weng B, Wang J J, Michaud F, et al. Atrial fibrillation detection using stationary wavelet transform analysis[C]//Engineering in Medicine and Biology Society, 2008. EMBS 2008. 30th Annual International Conference of the IEEE. IEEE, 2008: 1128-1131.

[8] Stridh M, Sornmo L, Meurling C J, et al. Sequential characterization of atrial tachyarrhythmias based on ECG time-frequency analysis[J]. IEEE Transactions on Biomedical Engineering, 2004, 51(1): 100-114.

[9] Goldberger A L, Amaral L A N, Glass L, et al. Physiobank, physiotoolkit, and physionet[J]. Circulation, 2000, 101(23): e215-e220.

[10] Jia Y, Shelhamer E, Donahue J, et al. Caffe: Convolutional architecture for fast feature embedding[C]//Proceedings of the 22nd ACM international conference on Multimedia. ACM, 2014: 675-
678.

[11] Huang C, Ye S, Chen $H$, et al. A novel method for detection of the transition between atrial fibrillation and sinus rhythm[J]. IEEE Transactions on Biomedical Engineering, 2011, 58(4): 1113-1119.

[12] Lee J, Reyes B A, McManus D D, et al. Atrial fibrillation detection using an iPhone 4S[J]. IEEE Transactions on Biomedical Engineering, 2013, 60(1): 203-206.

[13] Babaeizadeh S, Gregg R E, Helfenbein E D, et al. Improvements in atrial fibrillation detection for real-time monitoring[J]. Journal of Electrocardiology, 2009, 42(6): 522-526.

Address for correspondence.

Yong Xia

School of computer science and technology, Harbin Institute of Technology, Harbin, China

xiayong@hit.edu.cn 